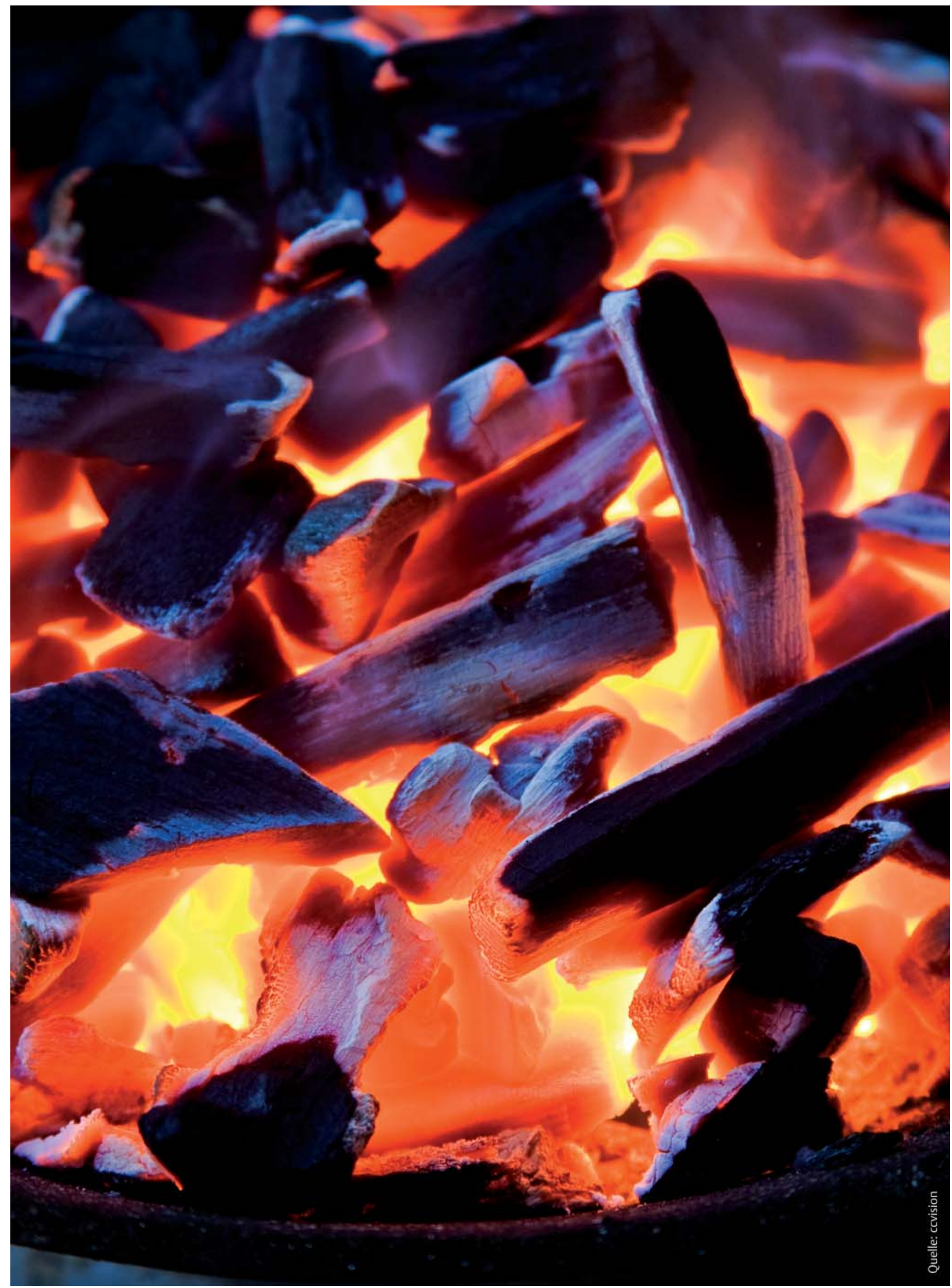




\section{Kohlenmonoxid: Unterschätzte Gefahr für Patienten und Retter}

Sven Thieme, Stefan Bosch

\section{Das Atemgift Kohlenmonoxid (CO) entsteht nicht nur bei unvollständigen Verbren- nungen. CO-Gefahren drohen auch bei unsachgemäß betriebenen Holzkohlegrills, beim Wasserpfeiferauchen oder bei der Lagerung von Holzpellets. Dabei können chronische oder akute Vergiftungen entstehen, die auch das Rettungspersonal ge- fährden. Dieser Beitrag beschreibt Symptomatik und Therapie der CO-Vergiftung sowie einsatztaktische Aspekte in CO-belasteten Räumen.}

\section{CO: Eigenschaften und Wirkung}

Kohlenmonoxid (CO) ist ein toxisch wirkendes Gas. Es entsteht bei unvollständiger Verbrennung und kann akute, oft tödliche, aber auch leichte, nicht sofort erkennbare Vergiftungen verursachen.

Menschen nehmen $\mathrm{CO}$ nicht wahr, denn es ist farb- und geruchlos. Da es leichter als Luft ist, verteilt es sich aufgrund seiner Diffusionsfähigkeit ungleich in Räumen. Während Kohlendioxid $\left(\mathrm{CO}_{2}\right)$ schwerer als Luft ist und sich am Boden sammelt, kann CO Wände und Decken durchdringen und sich über Etagen verteilen („Sickergas"). In unbelüfteten Räumen besteht durch CO Explosionsgefahr.

Beim Einatmen gelangt CO über die Lunge zu den roten Blutkörperchen. Für die Giftwirkung von $\mathrm{CO}$ sind drei Mechanismen verantwortlich $[1,17,18]$ :

1. CO verdrängt den Sauerstoff aufgrund seiner 200 300-fach höheren Affinität zum zweiwertigen Eisen des Hämoglobins in den Erythrozyten und bildet statt $\mathrm{O}-\mathrm{Hb}$ das stabilere Carboxy-Hämoglobin (CO-Hb).

2. $\mathrm{CO}$ bindet das noch vorhandene $\mathrm{O}_{2}$ stärker an das Hämoglobin, was die $\mathrm{O}_{2}$-Abgabe an das Gewebe vermindert und einer Linksverschiebung der Sauerstoffbindungskurve entspricht (Haldane-Effekt).

3. CO beeinträchtigt die Funktion wichtiger Enzyme in den Zellen und führt zu einem zellschädigenden Sauerstoffmangel (Hypoxie).

Merke

Das farb- und geruchslose Gas CO kann sich durch Decken und Wände ausbreiten und im Blut Sauerstoff aus den roten Blutkörperchen verdrängen.

\section{Symptomatik der CO-Vergiftung}

Die Symptome einer CO-Vergiftung können sehr variabel sein. Sie hängen vom $\mathrm{CO}-\mathrm{Hb}$-Gehalt des Blutes, der Expositionsdauer und der individuellen Empfindlichkeit des Patienten sowie seinen Vorerkrankungen ab. Deshalb müssen die folgenden Symptome nicht zwingend mit den gemessenen $\mathrm{CO}-\mathrm{Hb}$-Werten korrelieren.

- Leichte Vergiftungen (ab ca. 10-15\% CO-Hb-Gehalt im Blut)

Die Betroffenen klagen über Stirn- und Schläfenkopfschmerzen, Sehstörungen, Kurzatmigkeit, Herzsensationen und Schwindel.

- Mittelschwere Intoxikationen (30-40\% CO-HbGehalt)

Die Betroffenen können reizbar, müde, verwirrt oder erregt sein und ihre Urteils- und Entschlussfähigkeit ist beeinträchtigt, so dass sie sich selbst nicht mehr retten können. Weiterhin wurden beschrieben: Schwäche, Konzentrationsstörungen, optische und akustische Sensationen, Schmerzen in den Extremitäten, pathologische Reflexe und Pupillenreaktionen sowie Krampfanfälle. Ein Anstieg oder Abfall des Blutdrucks sowie Tachykardien können ebenso auftreten wie eine unregelmäßige oder oberflächliche Atmung im Wechsel mit Hyperventilation. Wegen Übelkeit und Erbrechens besteht Aspirationsgefahr. Zyanosezeichen können auftreten, und die lehrbuchmäßige kirschrote Hautfarbe zeigt sich nicht immer [17, 19]. Kinder weisen ein eher blasses Hautkolorit auf.

- Schwere Intoxikationen (ab 50 \% CO-Hb-Gehalt) Es treten unmittelbar Bewusstlosigkeit, Koma, Hyperthermie und Tod durch Atemlähmung oder Herzversagen mit Lungenödem ein.

\section{Merke}

Vergiftungen mit CO können je nach Konzentration in der Luft, Expositionsdauer und individueller Empfindlichkeit unterschiedliche Symptome hervorrufen. 
Unklare neurologische und kardiale Symptome sollten immer auch an eine CO-Intoxikation denken lassen.

\section{Differenzialdiagnostische Erwägungen}

Zunehmend wird auf die Gefahr chronischer CO-Expositionen hingewiesen, die mit neurologischen Symptomen einhergehen können $[15,16]$. Diese treten abhängig von der Expositionsdauer und der erreichten Serumkonzentration vor allem an den hypoxieempfindlichen Zielorganen ZNS, Herz, Lunge und Haut in Erscheinung. Besonders kardiopulmonal vorgeschädigte und anämische Patienten sind schneller betroffen [17]. Rauchgasinhalationen sind fast immer auch mit einer CO-Intoxikation verbunden, das darf nicht übersehen werden [20]. Zu beachten sind auch CO-Einflüsse bei alkoholisierten Patienten [21] und bei dunkelhäutigen Menschen, bei denen wegweisende Änderungen der Hautfarbe schwer erkennbar sind [19]. Auch bei Leichenschauen werden CO-Intoxikationen leicht übersehen bzw. nicht bedacht [19].

\section{Sauerstoff als Mittel der Wahl}

Die Notfalltherapie besteht in der Rettung des Betroffenen aus der CO-haltigen Atmosphäre (unter Beachten des Eigenschutzes) an die frische Luft. Die wichtigste therapeutische Maßnahme ist $\mathrm{O}_{2}$-Gabe, die den $\mathrm{CO}$ Gehalt des Blutes um 30-50\% pro Stunde senkt [25]. Auch nach Wiedererlangen des Bewusstseins soll weiterhin $\mathrm{O}_{2}$ gegeben werden. Je nach Schweregrad kann eine Intubation und Überdruckbeatmung notwendig sein, zumindest bis ein Blut-CO-Hb unter $20 \%$ erreicht ist [25]. In schweren Fällen ist eine hyperbare Oxygenation in einer Druckkammer notwendig.

Die Vitalfunktionen müssen überwacht und Störungen von Blutdruck, Herzfrequenz, Herzrhythmus und Säure-Basen-Haushalt therapiert werden. Folgende Wechselwirkungen sind zu bedenken: eine die Toxizität steigernde Wirkung der Katecholamine sowie die ZNSdämpfende Wirkung von Sedativa [25]. Das wichtigste "Gegengift“ ist $\mathrm{O}_{2}$. Übliche Antidota wie Thionin (Kataly$\sin ^{\circledR}$ ), Methylthionin, Hydroxocobalamin (Cyanokit ${ }^{\circledR}$ ) etc. haben keinen wesentlichen Einfluss auf CO-Vergiftungen und bedeuten nur unnötigen Zeitverlust [25].

Merke

Die wichtigste Maßnahme bei einer CO-Vergiftung ist die großzügige Gabe von Sauerstoff.

\section{Technische Tücken bei der Diagnostik}

Neben einer verwirrenden Symptomvielfalt bestehen bei CO-Vergiftungen zusätzlich technische Schwierigkeiten:
Konventionelle Pulsoxymeter erkennen kein $\mathrm{CO}-\mathrm{Hb}$ und zeigen vermeintlich normale $\mathrm{SpO}_{2}$-Werte ( $\mathbf{A} \mathbf{b b} \mathbf{b} \mathbf{1}$ ). $\mathrm{CO}-\mathrm{Hb}$ absorbiert Licht in einer ähnlichen Wellenlänge wie oxygeniertes Hämoglobin, so dass $\mathrm{CO}-\mathrm{Hb}$ fälschlicherweise als O-Hb gewertet wird. Deshalb dürfen bei Rauchgas-Verletzten normale Sättigungswerte im Pulsoxymeter nicht darüber hinwegtäuschen, dass dennoch eine relevante $\mathrm{CO}$-Vergiftung vorliegen kann und die Patienten dringend $\mathrm{O}_{2}$ benötigen.

Zur Bestätigung einer CO-Vergiftung ist eine frühzeitige Blutentnahme notwendig. Da im Rettungsdienst nicht immer Abnahmeröhrchen für eine Blutgasanalyse mitgeführt werden ( $\mathbf{A} \mathbf{A b}$ b. 2), kann zur Blutentnahme ersatzweise eine $2 \mathrm{ml}$-Spritze verwendet werden, die mit Heparin benetzt wurde. Dazu wird der Inhalt einer Heparin-Ampulle in eine $2 \mathrm{ml}$-Spritze aufgezogen und wieder ausgespritzt, so dass Konus und Spritzenwände benetzt sind. In der Klinik sollte diese Probe umgehend in einem Blutgasanalysegerät untersucht werden.

Nicht verwirren lassen sollte man sich durch Messwerte und Einheiten. Konzentrationen der Gase werden in Warn- und Messgeräten in ppm (parts per million, Teile einer Million bzw. millionster Teil) angegeben. Pulsoxymeter dagegen zeigen den Anteil von mit Sauerstoff beladenem Hämoglobin in Prozentwerten an. Da konventionelle Pulsoxymeter mit $\mathrm{CO}$ beladenes $\mathrm{Hb}$ jedoch nicht erkennen, benötigt man spezielle Pulsoxymeter. Diese bestimmen mithilfe eines separaten Messverfahrens im Absorptionsmaximum von $\mathrm{CO}-\mathrm{Hb}$, wie viel Prozent $\mathrm{Hb}$ mit $\mathrm{CO}$ beladen sind. In der Blutgasanalyse ( $\triangleright$ Abb. 2) werden die Anteile von $\mathrm{CO}-\mathrm{Hb}$ und $\mathrm{O}-\mathrm{Hb}$ ebenfalls in Prozentwerten angegeben.

Merke

Konventionelle Pulsoxymeter können den lebensbedrohlichen Sauerstoffmangel bei einer CO-Intoxikation aus messtechnischen Gründen nicht erkennen. Sie zeigen fälschlicherweise Normalwerte an.

\section{CO-Quellen im Haushalt}

Seitdem Menschen das Feuer nutzen, ist die Giftwirkung von CO bekannt. Über die verschiedenen CO-Quellen und deren gesundheitsschädliche Auswirkungen wurde immer wieder berichtet. Abgesehen vom klassischen Brandereignis treten in jüngster Zeit neben schweren akuten vermehrt auch leichte und chronische Intoxikationen in den Vordergrund, die im häuslichen Umfeld auftreten können [26]. Mit einem handelsüblichen Warngerät lassen sich im Haushalt gesundheitsgefährdende CO-Konzentrationen nachweisen: Beim Anfeuern eines Kamin- oder Kachelofens kann der erste Rauch kurzzeitig CO-Konzentrationen bis 300 ppm und mehr enthalten ( $\triangleright$ Abb.3). Sofern dieser Rauch in geringen Mengen in 
gut belüftete Räume austritt, dürfte die Belastung nicht relevant sein. Bei gestörter Kaminfunktion können jedoch schnell gefährliche Konzentrationen erreicht werden.

\section{FALLBEISPIEL}

\section{CO-Intoxikation beim Kaffeekochen}

Der weltberühmte isländische Schriftsteller Gunnar Gunnarsson beschreibt in seiner Novelle „Advent im Hochgebirge“ eine klassische $\mathrm{CO}$ - und $\mathrm{CO}_{2}$-Mischintoxikation mit ihren pulmonalen und neurologischen Symptomen. Der Schafhirte Benedikt wird in seiner Hütte im Hochland derart eingeschneit, dass die Belüftung beeinträchtigt ist: „In demselben Augenblick saß Benedikt im Finstern [Kerze erlischt infolge $\left.\mathrm{CO}_{2}\right]$.... Es war eine höchst unnatürliche Dunkelheit, die geradezu in den Augen brannte und einen am Halse packte und erwürgen wollte. Und zugleich war sie so freundlich, sie lockte einen zu schlafen - nur umzusinken und zu schlafen. ... Er versuchte sich zurechtzufinden, sich zu sammeln, zu denken. ... Sie sollten wohl hier unten ersticken? Da sollte doch gleich ...! Benedikt erhob sich, so schwer es ihm auch fiel, die Schlaftrunkenheit abzuschütteln; er schwankte zur Luke, stieß sie auf.“ [27]

\section{KOHLENMONOXIDQUELLEN}

nach [1 - 6]

- Brandgase können erheblich CO enthalten

- Gasöfen, Heizungen, Durchlauferhitzer und Badezimmerthermen

- Abgase von Fahrzeugen mit Verbrennungsmotor

- Holzkohlegrills (bei Betrieb in geschlossenen Räumen ohne ausreichende Belüftung)

- Wasserpfeifenrauch

- CO emittierende Chemikalien in geschlossenen Räumen

- CO in Leuchtgas, Flüssiggas

- Explosions- und Sprenggase (Bergbau, Steinbrüche)

- Gaswerke, Kokereien, Gichtgase von Hochöfen

- Abbeizmittel Methylenchlorid

- Holzpelletlager

\section{Merke}

CO wird bei allen Verbrennungsvorgängen mit unvollständiger Verbrennung von Kohlenstoff freigesetzt.

\section{Wasserpfeifen und Tischgrills}

Die unsachgemäße Benutzung von Holzkohlegrills in unzureichend belüfteten Räumen führt immer wieder

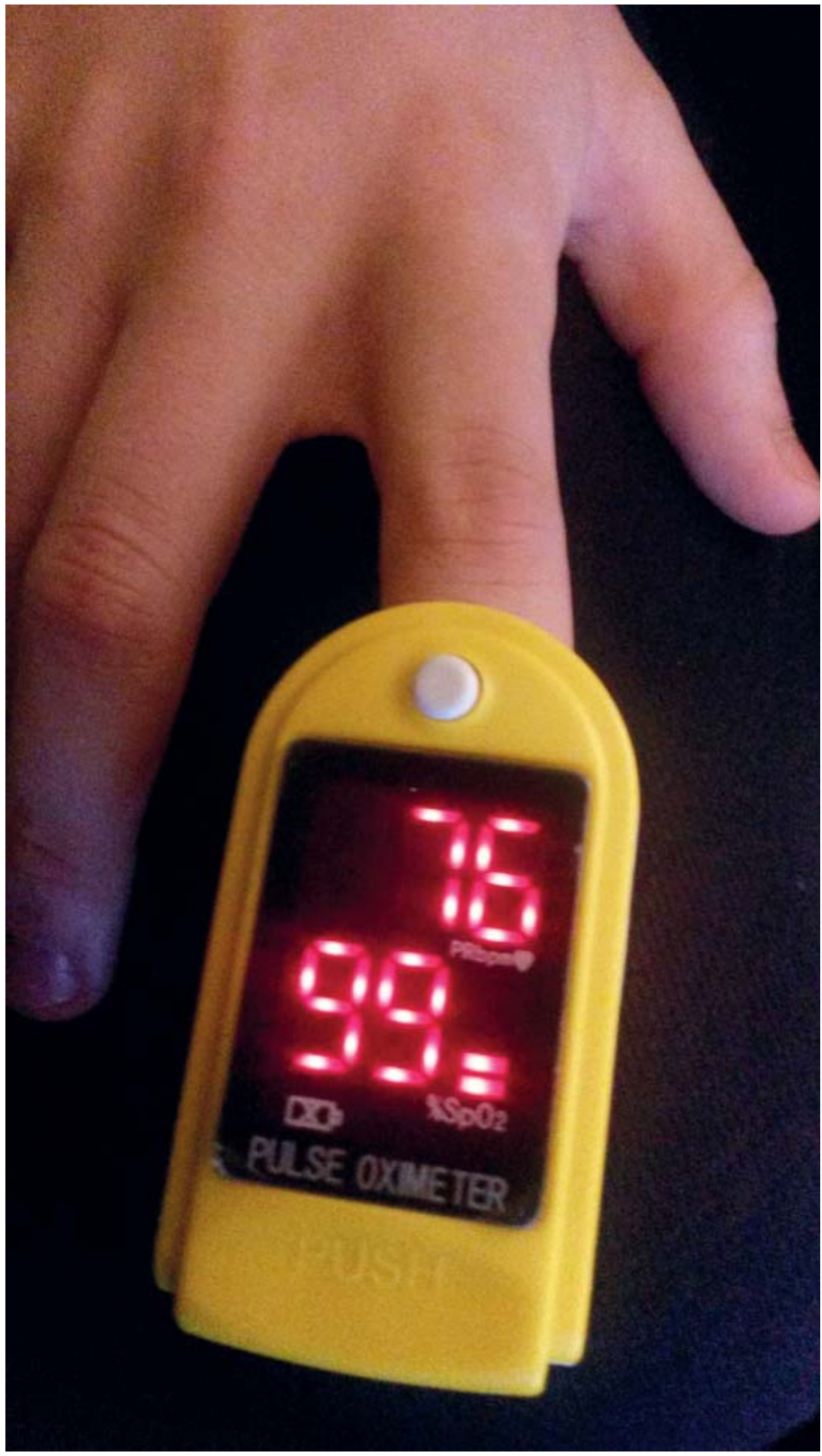

- Abb. 1 Normale Pulsoxymeter können eine CO-Vergiftung nicht erkennen und zeigen fälschlicherweise normale Messwerte an.

zu schweren Vergiftungen oft ganzer Familien oder Gruppen. In den letzten Jahren wurden vermehrt Suizidversuche mit Verbrennungsgasen aus Holzkohlegrills unternommen, die in geschlossenen oder gezielt abgedichteten Räumen angezündet wurden [5].

Zu CO-Vergiftungen kommt es bei jungen Menschen durch den populären Genuss von Wasserpfeifen. Mit $8-10 \%$ sind die CO-Hb-Konzentrationen von Rauchern gegenüber Nichtrauchern (4-5\%) ohnehin erhöht. Wasserpfeifen werden zu Unrecht als die harmlosere und gesündere Alternative zu Zigaretten betrachtet. Der Wasserpfeifenrauch enthält neben Nikotin und Teer 


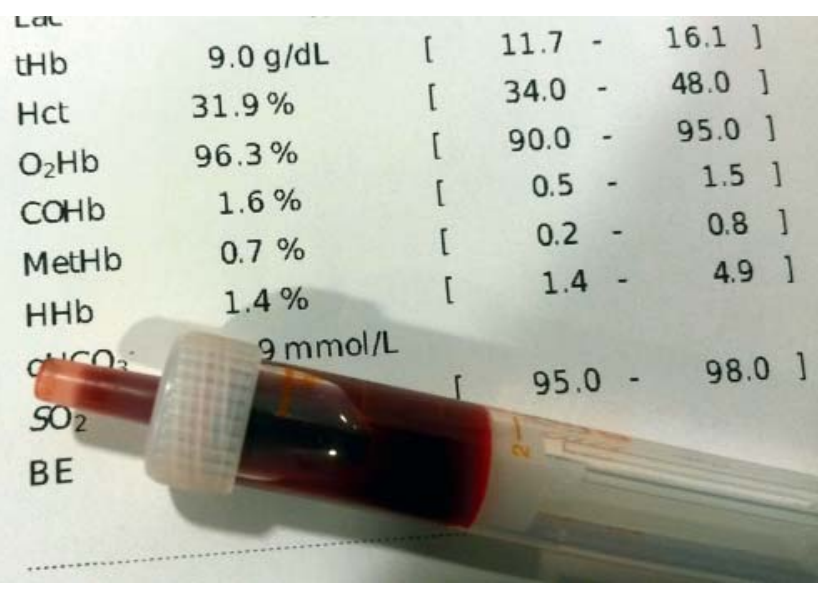

- Abb. 2 Abnahmeröhrchen für Blutgasanalyse mit Ausdruck der Messergebnisse.

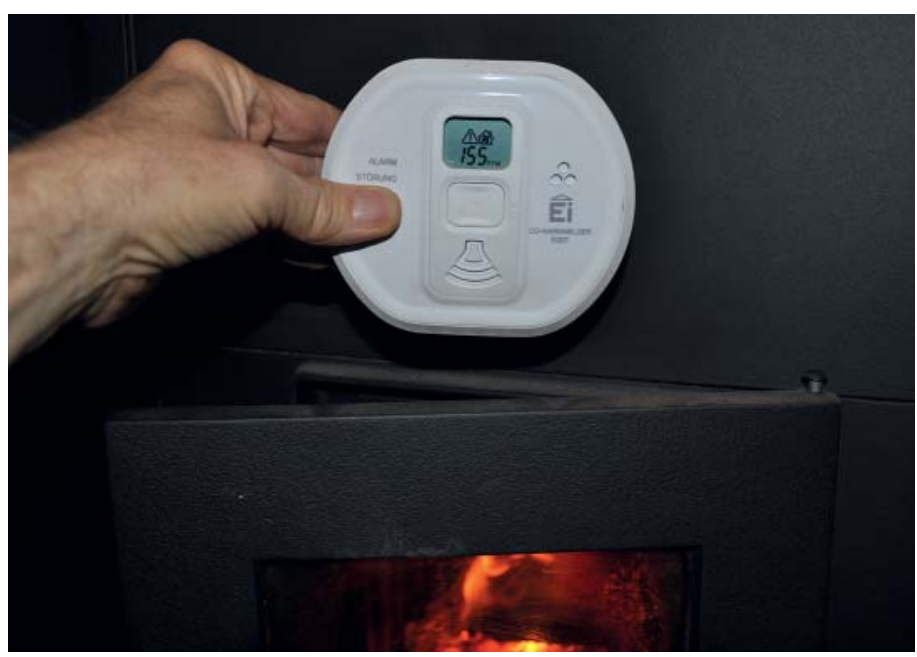

- Abb. 3 Beim Anfeuern lassen sich an der offenen Ofentür mit einem handelsüblichen CO-Warngerät kurzzeitig deutlich erhöhte CO-Konzentrationen nachweisen.

auch erhebliche CO-Mengen. Dabei spielt der Faktor Zeit eine Rolle: Aufgrund der längeren Inhalationsdauer wird bis zu zehnmal mehr CO als beim Zigarettenrauchen aufgenommen. Man schätzt weltweit 100 Millionen Wasserpfeifenraucher und damit eine entsprechend hohe Dunkelziffer an CO-Vergiftungen, die entweder chronisch verlaufen oder auch akute neurologische Symptome bis hin zu Bewusstseinsstörungen hervorrufen. Spätfolgen wie Gedächtnisstörungen, Ängste und Depressionen sind möglich. Mitunter finden sich erhöhte CO-Werte ohne entsprechende Symptomatik.

\section{FALLBEISPIEL}

\section{Familiengrillen im Gartenhaus}

Wegen Regenfällen verlegt eine Großfamilie ihr sonntägliches Grillfest in die kleine, schlecht belüfte- te Gartenhütte. Dort wird der Holzkohlegrill in Betrieb genommen. Nach einiger Zeit klagen mehrere Familienmitglieder über Kopfschmerzen, Schwindel und schwere Atemnot. Manche Betroffene zeigen eine rosige Gesichtsfarbe. Der alarmierte Rettungsdienst versorgt die Familie mit der Gabe von Sauerstoff über Reservoirmaske bzw. Nasensonde. Zwei Erwachsene mit schwerer Atemnot werden in die nächstgelegene Intensivstation aufgenommen und wegen hoher CO-Konzentrationen in eine Klinik mit Druckkammer zur hyperbaren $\mathrm{O}_{2}$-Therapie verlegt.

\section{FALLBEISPIEL}

\section{Bewusstlos nach Wasserpfeife}

Der Rettungsdienst wird zu einer 18-jährigen bewusstlosen Frau gerufen. Die kreislaufstabile Patientin wird mit GCS 3 in eine Klinik gebracht. In der Anamnese finden sich keine Hinweise auf Epilepsie, Drogen- oder Alkoholkonsum. Nach 15 min Bewusstlosigkeit erreicht die Patientin in der Notaufnahme einen GCS-Wert von 15 und ist beschwerdefrei. Auf Befragen berichtet sie vom Besuch einer Shisha-Bar, in der sie eine Stunde lang Wasserpfeife geraucht habe. Danach hatte sie starke Kopfschmerzen, Kribbelparästhesien, verschwommenes Sehen und ist schließlich kollabiert. In der Blutgasanalyse findet sich ein CO-Hb-Gehalt von $25 \%$. Nach $4 \mathrm{~h} \mathrm{O}$-Therapie mit Reservoirmaske und einem Flow $>10 \mathrm{I} / \mathrm{min}$ ist der Wert auf $7 \%$ gefallen. (Kasuistik nach [6])

\section{Todesfälle durch CO aus Holzpellets}

Die Gefahr von CO-Emissionen aus Holzpellets ist Rettungsdienstmitarbeitern häufig nicht bekannt und bewusst, obwohl Holzpellets zunehmend als alternative Energieträger auch in Privathaushalten in größerer Menge gelagert werden. Im Jahr 2013 soll es in Deutschland 320000 Pelletheizanlagen mit einem Lagervolumen von 2,3 Millionen Tonnen gegeben haben.

Erste Hinweise auf CO-Ausgasungen bei der Lagerung von Holzpellets wurden aus Schweden bekannt [7]. Auf Transportschiffen, die Pellets von Kanada nach Europa brachten, wurden in den Laderäumen und Treppengängen CO-Konzentrationen bis 14650 ppm gemessen. Neben $\mathrm{CO}$ wurden in Pelletlagern auch $\mathrm{CO}_{2}$, Methan, Butan, Ethylen, Propylen, Ethan und Aldehyde nachgewiesen [8]. CO, andere Gase und „volatile organische Komponenten “ (VOCs) entstehen in Holzpellets durch autooxidative Prozesse von Fett, ungesättigten Fettsäuren und Harzsäuren im Holz [2]. Das Ausmaß der CO-Emissionen ist unkalkulierbar, da es von vielen Faktoren abhängt, wie z. B. der verarbeiteten Holzart, von der 
- Tab. 1 CO-Messergebnisse an verschiedenen Messorten, klassiert nach den einsatztaktischen Empfehlungen nach [12].

\begin{tabular}{|c|c|c|c|c|c|}
\hline Literatur & Messort & $<30 \mathrm{ppm}$ & $\begin{array}{l}30-200 \mathrm{ppm} \\
\text { Aufmerksamkeitsschwelle }\end{array}$ & $\begin{array}{l}200-500 \text { ppm } \\
\text { Gefährdungs- } \\
\text { schwelle }\end{array}$ & $\begin{array}{l}>500 \text { ppm } \\
\text { Rückzugs- } \\
\text { schwelle }\end{array}$ \\
\hline [7] & Warenlager & & 56 & & \\
\hline [8] & Transportschiffe & & & & $1460-14650$ \\
\hline [13] & Schiffscontainer & & & & $\max .1502$ \\
\hline $\begin{array}{l}\text { eigene } \\
\text { Messungen }\end{array}$ & Gewebesilo 4,8t & & phasenweise bis 65 & & \\
\hline [14] & 2/3 aller Pelletlager & & $>30$ & & \\
\hline [14] & $9 \%$ aller Pelletlager & & & & $>1000$ \\
\hline [3] & Pelletlager $82 \mathrm{~m}^{3}$ & & & & $2000-7500$ \\
\hline [2] & $\begin{array}{l}\text { Pelletbunker, Todesfall un- } \\
\text { ter dem Verschlussdeckel }\end{array}$ & & & & 1000 \\
\hline [2] & $\begin{array}{l}\text { derselbe Bunker, in } 280 \mathrm{~cm} \\
\text { Tiefe }\end{array}$ & & & & 2500 \\
\hline [11] & Pelletlager Schrägboden & & & & $\max .717$ \\
\hline [11] & Pelletlager Silo & & & & $\max .614$ \\
\hline [11] & Pelletlager Gewebesilo & $\max .2$ & & & \\
\hline [11] & Kellerraum/kubisches Lager & & max. 60 & & \\
\hline [7] & Lager im Haushalt & & 123 & & \\
\hline
\end{tabular}

Raumtemperatur im Lager, der Sauerstoffverfügbarkeit und der Oberflächenausdehnung der Pellets sowie von deren Alter bzw. Lagerungsdauer [2, 3, 9-11]. Zwischen 2002 und 2012 kam es in Europa zu mindestens 14 dokumentierten und gesicherten CO-bedingten Todesfällen im Zusammenhang mit Holzpellets [3]. Ein tödlicher Fall bei Wartungsarbeiten in einem Pelletbunker wurde 2015 aus Deutschland berichtet [2].

\section{Merke}

Holzpellets können unter Umständen CO in gesundheitsgefährdenden Konzentrationen freisetzen. Auch in Privathaushalten ist mit einer CO-Belastung zu rechnen.

\section{CO aus Holzpellets im Hauskeller}

Die in Privathaushalten üblichen Gewebetanks können erfahrungsgemäß erhebliche CO-Mengen abgeben: Nach Befüllen mit 4,8 Tonnen frischen Pellets im Frühjahr gab das installierte CO-Warngerät wochenlang regelmäBig Alarm. Je nach Belüftung wurden Werte zwischen 30 und 65 ppm gemessen. Mit fortschreitender Lagerungsdauer blieben weitere Alarme aus. Die Werte liegen über der „Aufmerksamkeitsschwelle“ ( $\triangleright$ Tab. 1) und stellen bei längerer Exposition, wie z. B. mehrstündige Arbeiten im Keller, ein Gesundheitsrisiko dar. Die in - Tab. 1 aufgelisteten, in Holzpelletlagern ermittelten CO-Werte dokumentieren eindrücklich, dass der Großteil der Messergebnisse entweder im „Aufmerksamkeitsbereich“ oder über 500 ppm und damit über der „Rückzugs- schwelle“ lag. Auch wenn die Bedingungen in Pelletlagern unterschiedlich sind, zeigt die Tabelle, dass in solchen Lagern und ihrer Umgebung mit CO-Konzentrationen unterschiedlicher Höhe gerechnet werden muss, die sogar zum Rückzug zwingen können.

\section{FALLBEISPIEL}

\section{Tod im Pelletbunker}

Ein 43-jähriger Mann steigt ohne Schutzvorkehrungen zu Wartungsarbeiten in einen unterirdischen Pelletbunker. Einige Zeit später liegt er leblos bäuchlings auf der Pelletoberfläche. Eine weitere Person steigt ein, um nach ihm zu sehen. Sie hält die Luft an und verlässt den Bunker umgehend wieder, da der Mann offensichtlich tot ist. Die hinzugerufene Feuerwehr kann ihn nur noch tot bergen und misst am Bunkereingang - trotz geöffneter Luken - noch 1000 ppm CO, auf Höhe des Leichnams 2500 ppm. Bei der Obduktion finden sich hellrote Leichenflecke, Nagelbetten und Organverfärbungen, kirschrotes Blut und Pelletpartikel in den Atemwegen. (Kasuistik nach [2])

\section{Rettungsdienstliche Aspekte}

Holzpellets können aufgrund diverser Faktoren $\mathrm{CO}$ in unterschiedlich hohen Konzentrationen abgeben ( $\triangleright$ Tab. 1). Selbst kleine Lager in Privathaushalten haben für den Rettungsdienst in mehrfacher Hinsicht Bedeutung: Je 
- Tab. 2 Empfehlungen zum einsatztaktischen Vorgehen bei der Notfallversorgung in CO-haltiger Umgebung (Zusammenfassung nach [12, 23]).

\begin{tabular}{|c|c|c|}
\hline CO-Konzentration in ppm & Klinisch-toxikologische Risikobewertung & Empfohlenes Verhalten im Rettungsdiensteinsatz \\
\hline$<30$ ppm & Arbeitsplatzgrenzwert & Keine Gefährdung \\
\hline 33 ppm & Einsatztoleranzwert & für ungeschütztes Rettungspersonal \\
\hline $30-200$ ppm & Aufmerksamkeitsschwelle & $\begin{array}{l}\text { " zügig versorgen } \\
\text { " gut lüften } \\
\text { " ggf. Fachkräfte hinzuziehen }\end{array}$ \\
\hline$\geq 60 \mathrm{ppm}$ & $\begin{array}{l}\text { Feuerwehr: } \\
\text { Rückzugsempfehlung [23] }\end{array}$ & Zugang nur mit umluftunabhängigem Atemschutzgerät \\
\hline $60 \mathrm{ppm}$ & $\begin{array}{l}\text { Kurzzeitgrenzwert } \\
\text { Keine Gefährdung für ungeschütztes Ret- } \\
\text { tungspersonal bei Exposition < } 1 \text { Stunde }\end{array}$ & $\begin{array}{l}\text { - Medizinische Versorgung ohne Unterbrechung durchführen } \\
\text { - Raumlüftung: Fenster und Türen öffnen } \\
\text { - CO-Quelle identifizieren, weitere Freisetzung verhindern, } \\
\text { wenn nicht möglich, Fachkräfte (Feuerwehr, Schornsteinfeger, } \\
\text { Störungsdienst) informieren } \\
\text { - bei potenziell Exponierten Schnelldiagnostik mit CO-Hb-Pulsoxy- } \\
\text { meter oder Blutentnahme für CO-Hb-Bestimmung in der Klinik }\end{array}$ \\
\hline $200-500$ ppm & $\begin{array}{l}\text { Gefährdungsschwelle } \\
\text { nach } 30 \text { min leichte Vergiftungssymptome } \\
\text { möglich }\end{array}$ & $\begin{array}{l}\text { - erst belüften } \\
\text { - erst retten, dann versorgen } \\
\text { - Feuerwehr rufen } \\
\text { " zuerst Belüftungsmaßnahmen ergreifen } \\
\text { " wenn effektive Belüftung nicht möglich, Patienten aus Gefah- } \\
\text { renbereich bringen } \\
\text { - dann medizinische Versorgung } \\
\text { - } \text { Aufenthaltsdauer im Gefahrenbereich minimieren } \\
\text { - Feuerwehr alarmieren }\end{array}$ \\
\hline 500 ppm & $\begin{array}{l}\text { Messbereichsgrenze vieler CO-Warngeräte! } \\
\text { Vergiftungssymptome möglich } \\
\text { " nach } 10 \text { min Exposition leicht } \\
\text { " nach } 30 \text { min mittelschwer }\end{array}$ & \\
\hline$>500 \mathrm{ppm}$ & Rückzugsschwelle & $\begin{array}{l}\text { - Zurückziehen ohne Versorgung } \\
\text { - Feuerwehr rufen, Personenrettung nur mit Atemschutz } \\
\text { " Gefahrenbereich verlassen } \\
\text { - Feuerwehr alarmieren } \\
\text { - Personenrettung nach Absenken der CO-Konzentration oder } \\
\text { unter umluftunabhängigem Atemschutz }\end{array}$ \\
\hline 1000 ppm & $\begin{array}{l}\text { Vergiftungssymptome möglich } \\
\text { " nach wenigen Minuten mittelschwer } \\
\text { " potenziell tödlich nach Stunden }\end{array}$ & \\
\hline 3000 ppm & $\begin{array}{l}\text { Vergiftungssymptome möglich } \\
\text { " nach wenigen Minuten schwer } \\
\text { " potenziell tödlich nach ca. } 30 \text { min }\end{array}$ & \\
\hline 10000 ppm & " potenziell tödlich innerhalb weniger Minuten & \\
\hline
\end{tabular}

nach Lagerung (Silo, Gewebetank, Schrägboden) können durch lose Materialmassen Personen verschüttet werden. Durch Pelletabrieb entsteht eine staubhaltige explosionsgefährdete Atmosphäre. Alle aufgeführten Risiken betreffen Eigenschutz, Einsatztaktik und differenzialdiagnostische Erwägungen.

\section{Eigenschutz und Einsatztaktik}

Rettungspersonal ist gegen CO-Einflüsse nicht geschützt. Deshalb werden zunehmend CO-Warngeräte mitgeführt, um eine Gefahr frühzeitig zu erkennen. Bei der Feuerwehr wird bereits für 60 ppm eine klare Empfehlung zum Rückzug ausgesprochen und nur ein Zugang mit umluftunabhängigen Atemschutzgeräten empfohlen [23, 24]. Ein abgestuftes und situationsadaptiertes Vorgehen trägt dazu bei, dem Dilemma einer verzögerten Patientenversorgung zu entgehen. Sinnvolle Strategien, an denen sich das Rettungspersonal mithilfe von CO-Warngeräten orientieren kann, sind in $>$ Tab. 2 zusammengefasst [12]. Bestimmende Faktoren sind dabei die vorhandene CO-Konzentration und die Aufenthaltsdauer in der jeweiligen Atmosphäre. 


\section{Merke}

Um weder das Rettungspersonal zu gefährden noch dem Patienten eine Notfallbehandlung unnötig lange vorzuenthalten, wurden Grenzwerte empfohlen, bei denen eine kurzzeitige Exposition des Rettungspersonals während der Erstversorgung als vertretbar angesehen wird.

\section{Hinweise für den Notarzt- und Rettungsdienst}

Für den Rettungsdienst ergeben sich folgende Empfehlungen im Hinblick auf mögliche CO-Expositionen im Einsatz:

\section{... bezüglich Pelletlagern}

Bei Herstellung, Lagerung und Transport, aber auch bei Lagerung in Privathaushalten ist mit CO-Ausgasungen aus Holzpellets zu rechnen. Teilweise werden CO-Konzentrationen erreicht, die ungeschütztes Rettungspersonal zum Rückzug zwingen ( $C O$ > 500 ppm). Eine Reihe tragischer Todesfälle unterstreicht dies deutlich.

Daher wird der konsequente Einsatz von CO-Warngeräten bei Rettungseinsätzen empfohlen. Sie ermöglichen aufgrund ihrer Messergebnisse ein differenziertes Vorgehen und verhindern so, dass einerseits Rettungspersonal gefährdet und andererseits Patienten aus übertriebener Vorsicht verzögert versorgt werden.

CO-Warngeräte ( $\triangleright$ Abb.4) werden für jedes Rettungsmittel empfohlen [1, 24]. Sie nützen jedoch nur, wenn sie im Einsatz immer konsequent am Körper getragen werden. Da CO-Gefahren auch drohen, wenn es am Einsatzort nicht brennt, sollten die Geräte bei jedem Einsatz mitgeführt werden. Auch die Befestigung am Rucksack ist ungünstig: Wird er abgestellt und die Helfer dringen weiter vor, haben sie kein Warngerät mehr.

CO wird nur dann sicher erkannt, wenn die Geräte offen zugänglich am Körper und nicht in der Brusttasche oder von der Jacke bedeckt getragen werden. Bauartbedingt warnen CO-Warngeräte bei unterschiedlichen Schwellen bzw. Grenzwerten. Über diese Grenzen muss man sich vor dem Einsatz informieren, um richtig handeln zu können.

CO-Warngeräte liefern wichtige differenzialdiagnostische Hinweise, um Symptome richtig zu deuten.

Warnungen vor CO - wie Schilder an Lagerräumen oder stationäre Warngeräte - niemals ignorieren [22], sondern auf Eigenschutz achten und ggf. kompetente Fachhilfe nachfordern.

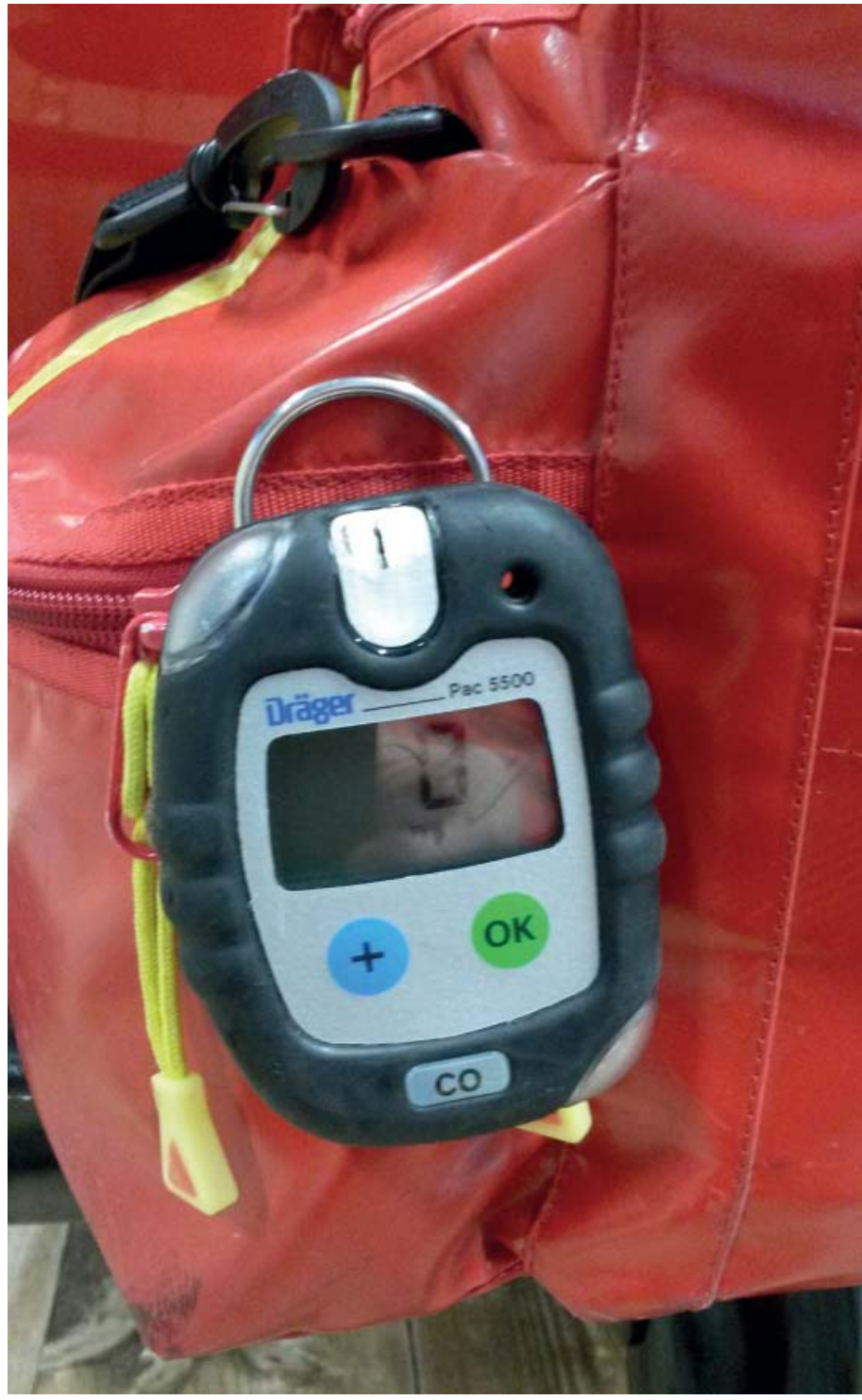

- Abb.4 CO-Warngerät für Rettungsdienst und Feuerwehr. (Das Warngerät ist hier jedoch ungünstig positioniert. Siehe Details zur Positionierung etc. im Text).

Je nach Ausführung (Silo, Gewebetank, Schrägbodenlager) besteht in Pelletlagern Rutsch-, Verschüttungs- und staubbedingte Explosionsgefahr.

Merke

CO-Warngeräte im Rettungsdienst helfen nur, wenn sie immer konsequent und „offen“ am Körper getragen werden.

\section{... bezüglich CO-Expositionen}

Bereits bei der Notrufabfrage in der Leitstelle können sich Hinweise auf CO-Gefahren ergeben, z. B. „bewusstlose Person hinter verschlossener Tür/im Keller/im Pelletlager“ [23], was die gleichzeitige Alarmierung entsprechender Rettungskräfte erfordert. 
Bei Verdacht auf eine Intoxikation sollen CO-Messungen durchgeführt werden. Dies kann präklinisch mit CO-Hbdetektierenden Pulsoxymetern oder nachträglich in der Klinik mit einem Blutgasanalysegerät aus vor Ort entnommenen, heparinisierten Blutproben erfolgen. Konventionelle Pulsoxymeter erkennen $\mathrm{CO}-\mathrm{Hb}$ nicht und zeigen vermeintlich normale $\mathrm{SpO}_{2}$-Werte.

Bei unklaren neurologischen oder kardialen Symptomen sollte CO immer differenzialdiagnostisch erwogen und die Anamnese auf mögliche Expositionen gelenkt werden (Pelletlager? Längerer Aufenthalt in oder in der Nähe von CO-Quellen?; siehe Box).

\section{KERNAUSSAGEN}

- CO ist ein farb- und geruchloses Atemgift, das leichter ist als Luft und durch Wände und Decken diffundiert.

- Es entsteht bei allen unvollständigen Verbrennungsvorgängen, aber auch durch andere Quellen, bspw. bei der Lagerung von Holzpellets oder in Wasserpfeifen.

- Erste Symptome einer CO-Intoxikation sind: Kopfschmerzen, Sehstörungen, Schwindel und Kurzatmigkeit.

- Konventionelle Pulsoxymeter sind zum Nachweis einer CO-Intoxikation ungeeignet.

- Das Mittel der Wahl bei einer CO-Vergiftung ist sofortige, hochdosierte Gabe von Sauerstoff.

- Rettungspersonal sollte im Einsatz immer COWarngeräte offen am Körper tragen.

\section{Interessenkonflikt}

Die Autoren geben an, dass kein Interessenkonflikt besteht.

\section{Über die Autoren}
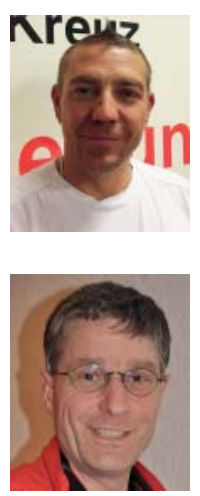

\section{Sven Thieme}

ist Notfallsanitäter und Praxisanleiter beim DRK Pforzheim, Rettungswache Mühlacker, und Zugführer bei der Freiwilligen Feuerwehr Maulbronn sowie Kreisausbilder für Truppmann und Truppführer.

\section{Dr. Stefan Bosch}

ist seit fast 40 Jahren im Rettungsdienst tätig und arbeitet als Facharzt für Anästhesiologie und als Notfallmediziner an den Enzkreiskliniken in Mühlacker.

\section{Korrespondenzadresse}

\section{Dr. Stefan Bosch}

Facharzt für Anästhesiologie

Notfallmedizin, Akupunktur, Betriebsmedizin

75447 Sternenfels

Metterstr. 16

stefan-bosch@web.de

\section{Literatur}

[1] Kaiser G, Schaper A. Akute Kohlenmonoxydvergiftung. Ein alter Hut in neuen Schachteln. Notfall Rettungsmed 2012; 15: 429-435

[2] Cortis ], Bender K, Rothschild MA et al. Tödliche Holzpellets. Kohlenstoffmonoxidintoxikation durch unsachgemäße Lagerung. Rechtsmedizin 2015; 25: 101 - 106

[3] Gauthier S, Grass H, Lory M et al. Lethal Carbon Monoxide Poisoning in Wood Pellet Storerooms - Two Cases and a Review of the Literature. Ann Occup Hyg 2012; 56: 755 - 763

[4] Wagner F, Henze C, Baumann $\mathrm{H}$ et al. Vom Badezimmer in die Druckkammer. Notfall Rettungsmed 2013; 16: 377-381

[5] Püschel K. Holzkohlegrill und Kohlenmonoxid. Tödliche Unfallgefahr und "neue“ Suizidmethode. Rechtsmedizin 2011; 21: $100-101$

[6] von Rappard J, Schönenberger M, Bärlocher L. Case report: Carbon monoxide poisoning following use of a water pipe/ hookah. Dtsch Arztebl 2014; Int 111: 674-679

[7] Svedberg U, Högberg H, Högberg J et al. Emission of Hexanal and Carbon Monoxide from Storage of Wood Pellets, a Potential Occupational and Domestic Health Hazard. Ann Occup Hyg 2004; 48: 339-349

[8] Svedberg U, Samuelsson J, Melin S. Hazardous Off-Gasing of Carbon Monoxide and Oxygen Depletion during Ocean Transportation of Wood Pellets. Ann Occup Hyg 2008; 52: 259 266

[9] Kuang X, Shankar TJ, Bi XT et al. Characterization and Kinetics Study of Off-Gas Emissions from Stored Wood Pellets. Ann Occup Hyg 2008; 52: 675-683

[10] Kuang X, Shankar T], Bi XT et al. Rate and peak concentrations of Off-Gas Emissions in Stored Wood Pellets - Sensitivities to Temperature, Relative Humidity, and Headspace Volume. Ann Occup Hyg 2009; 53: 789-796

[11] Spahn R. Abschlussbericht zur Sonderaktion „Lagerung von Holzpellets“ Februar-Mai 2013. Hrsg. Staatsministerium für Wirtschaft, Arbeit und Verkehr Freistaat Sachsen. 2013: 1-20

[12] Kaiser G, Müller D. Einsatz von Kohlenstoffmonoxidwarngeräten im Rettungsdienst. Hinweise und Empfehlungen zur Handhabung und Festlegung von Warnschwellen. Notfall Rettungsmed 2014; 17: 141 - 146

[13] Luyts L, Mück O. Container-Sicherheit auf Terminals in Benelux-Ländern: Praktische Erfahrungen. Zbl Arbeitsmed 2011; 61: $408-411$

[14] Emhofer W, Pointer C. Lagertechnik und Sicherheit bei der Pelletlagerung. Graz: Bioenergy 2020+. 2009

[15] Prockop LD, Chichkova RI. Carbon monoxide intoxication: An updated review. J Neurol Sciences 2007; 262: 122-130

[16] Weaver LK. Carbon Monoxide Poisoning. N Engl J Med 2009; 360: $1217-1225$

[17] Zilker T. Klinische Toxikologie für die Notfall- und Intensivmedizin. Uni-med Verlag. Bremen; 2008: 87-94 
[18] Blumenthal I. Carbon monoxide poisoning. J R Soc Med 2001; 94: $270-272$

[19] Guddat SS, Schalinski S, Matschke J et al. Tödliche Kohlenmonoxidintoxikation bei einem Schwarzafrikaner. Rechtsmedizin 2007; 17: 315-317

[20] Siemers F, Mailänder P. Inhalationstrauma, Kohlenmonoxidund Cyanidintoxikation. Notfall Rettungsmed 2010; 13: 17 22

[21] Schönemann B, Adam H]. Kohlenmonoxidvergiftung. Wichtige Differentialdiagnose bei Bewusstseinsstörungen. Notfall Rettungsmed 2009; 12: 379-382

[22] Franke M. Kohlenmonoxid im Pelletbunker. Die ungeahnte Gefahr. LSV kompakt. 2012: 6

[23] Deutscher Feuerwehrverband (Hrsg.). Rahmenempfehlungen zu Einsätzen bei Verdacht auf einen CO-Notfall innerhalb von Räumlichkeiten. Fachempfehlung Nr. 04/2012 vom 15. Mai 2012. Im Internet: http://www.feuerwehrverband.de/fe-conotfall.html Stand 2.4.2017

[24] Müller H, Schremmer R, Pfeuffer M. MMH-Studie „Gefährdung durch Kohlenstoffmonoxid an der Einsatzstelle“. Feuerwehr Wiesbaden (Hrsg.) 2012: 21

[25] Ludewig R, Regenthal R. Akute Vergiftungen und Arzneimittelüberdosierungen. 11. Aufl Stuttgart: Wissenschaftliche Verlagsgesellschaft Stuttgart; 2015: 384-388

[26] Bosch S. Tischgrills, Wasserpfeifen und Holzpellets: Unerwartete Kohlenmonoxidgefahren. Biol Unserer Zeit 2016; 46: $114-117$

[27] Gunnarson G. Advent im Hochgebirge. Stuttgart: Reclam. 2006: $46-47$

\section{Bibliografie}

DOI https://doi.org/10.1055/s-0042-124038 retten 2017; 6: 218-229

(c) Georg Thieme Verlag KG Stuttgart · New York ISSN 2193-2387 


\section{Punkte sammeln auf CEF, thieme.de}

口神回

Diese Fortbildungseinheit ist 12 Monate online für die Teilnahme verfügbar.

Sollten Sie Fragen zur Online-Teilnahme haben, finden Sie unter cme.thieme.de/hilfe

eine ausführliche Anleitung. Wir wünschen viel Erfolg beim Beantworten

der Fragen!

Unter https://eref.thieme.de/ZZWHQGA oder über den QR-Code kommen Sie direkt zum Artikel zur Eingabe der Antworten.

\section{Frage 1}

Um wie viel höher ist Affinität von Kohlenmonoxid (CO) zum Hämoglobin im Vergleich zu Sauerstoff $\left(\mathrm{O}_{2}\right)$ ?
A 5- bis 10-fach
B 20- bis 40-fach
C 96- bis 98-fach
D 200-bis 300-fach
E über 1000-fach

\section{Frage 2}

Welche drei Eigenschaften treffen auf CO zu?
A Farblos, stechender Geruch und leichter als Luft
B Farblos, geruchlos und leichter als Luft
C Farblos, geruchlos und schwerer als Luft
D Graue Farbe, geruchlos und schwerer als Luft
E Farblos, geruchlos und gleich schwer wie Luft

\section{Frage 3}

Was sind die ersten Symptome einer leichten CO-Vergiftung?

A Kopfschmerzen, Bewusstlosigkeit, Schwindel und Kurzatmigkeit

B Kopfschmerzen, Zyanose, Schwindel und Kurzatmigkeit

C Kopfschmerzen, Sehstörungen, Hörstörungen und Kurzatmigkeit

D Kopfschmerzen, Hörstörungen, kirschrote Haut und Husten

E Kopfschmerzen, Sehstörungen, Schwindel und Kurzatmigkeit

\section{Frage 4}

Welche Ursache kommt für die Entstehung von CO im Haushalt nicht infrage?
A Weinkeller mit Fässern
B Defekte Heizanlage im Keller
C Gasdurchlauferhitzer im Bad
D Holzpelletlager
E Abbeizmittel Methylenchlorid

\section{Frage 5}

Welche Einsatzstrategie wählen Sie bei einem CO-Wert von $\leq 200$ ppm auf Ihrem Gaswarngerät?

A Sofort lüften des Raums, zügiges Versorgen des Patienten, Fachkräfte nachfordern

B Sofortiger Rückzug, Nachfordern der Feuerwehr, Patientenrettung mit Atemschutz

C Sofortige Versorgung des Patienten, Fachkräfte nachfordern

D Sofortige Rettung aus dem Gefahrenbereich, dann Versorgung, Feuerwehr rufen

E Immer Feuerwehr vor Rettung und Versorgung rufen

\section{Frage 6}

Welche Einsatzstrategie wählen Sie bei einem CO-Wert von 300 ppm auf Ihrem Gaswarngerät in einem Pelletlager ohne Lüftungsmöglichkeit?

A Lüften über den Lagerzugang, zügiges Versorgen des Patienten, Fachkräfte nachfordern

B Sofortiger Rückzug, Nachfordern der Feuerwehr, Patientenrettung mit Atemschutz

C Sofortige Versorgung des Patienten, Fachkräfte nachfordern

D Sofortige Rettung aus dem Gefahrenbereich, dann Versorgung, Feuerwehr rufen

E Immer Feuerwehr vor Rettung und Versorgung rufen

\section{Frage 7}

Welches Medikament verabreichen Sie als erstes beim Verdacht auf einen CO-Intoxikation?
A Hydroxocobalamin (Cyanokit $\left.{ }^{\circledR}\right)$ i. v.
B Salbutamol inhalativ
C Hochdosiert Sauerstoff
D Obidoxim (Toxogonin $\left.{ }^{\circledR}\right)$ i. v.
E Natriumthiosulfat i.v.

\section{- Weitere Fragen auf der folgenden Seite...}




\section{Punkte sammeln auf CEEthieme.de}

\section{Fortsetzung ...}

\section{Frage 8}

Beschreiben Sie kurz eine alternative Möglichkeit zur Blutabnahme für eine Blutgasanalyse im Krankenhaus, wenn kein dafür vorgesehenes Abnahmeröhrchen vorhanden ist?
A Sterile $2 \mathrm{ml}-$ Spritze
B 2ml-Spritze, die „heparinisiert" wurde
C Abnahmeröhrchen für das Blutbild
D Abnahmeröhrchen für die Gerinnung
E Abnahmeröhrchen für Serum

\section{Frage 9}

Welche Gefahr besteht bei einer CO-Vergiftung hinsichtlich eines konventionellen Pulsoxymeters?

A Es zeigt $\mathrm{SaO}_{2}$-Werte an, die höher sind als die tatsächliche $\mathrm{O}_{2}$-Sättigung des Hämoglobins.

B Es zeigt $\mathrm{SaO}_{2}$-Werte an, die der tatsächlichen $\mathrm{O}_{2}$-Sättigung des Hämoglobins entsprechen.

C Es zeigt $\mathrm{SaO}_{2}$-Werte an, die niedriger als die tatsächliche $\mathrm{O}_{2}$-Sättigung des Hämoglobins liegen.

D Konventionelle Pulsoxymeter zeigen immer die Sättigung des Hämoglobins mit $\mathrm{O}_{2}$ und $\mathrm{CO}$ an.

E Mit konventionellen Pulsoxymetern lässt sich zuverlässig eine CO-Intoxikation erkennen.

\section{Frage 10}

Was sollte bei der Menschenrettung in Räumen mit sehr hoher CO-Konzentration getragen werden?
A Partikelfiltermaske
B Dicht schließende OP-Maske
C Sauerstoffmaske mit hohem Flow
D Umluftunabhängige Atemschutzgeräte
E Besondere Schutzmaßnahmen sind nicht erforderlich. 